\title{
Renal Dysplasia: A 6 Year Retrospective Study with Clinicopathological Correlation
}

\author{
Clement Wilfred Devadass*, Madhu Aramane Basavaraj, Prasanna Shetty Badila and Mangala Gouri \\ Dep’t of Pathology, MS Ramaiah Medical College and Hospitals, MSRIT Post, MSR Nagar, Bangalore (India)
}

\section{ABSTRACT}

Bakground: Renal dysplasia (RD) is rare congenital disease resulting from anomalous development of metanephric elements. The objective of the study was to evaluate the clinicopathologic features of RD with emphasis on the morphological changes.

Methods: A retrospective study of RD cases diagnosed on nephrectomy specimens over a duration of six years at a south Indian tertiary health care centre. The clinical, radiological and macroscopic features were retrieved and microscopic morphological features were reviewed.

Results: A total of 13 cases of RD were obtained, all of which were unilateral, with age range of 20 weeks of gestation to 54 years. Male predominance (61\%) was noted and left kidney was preponderantly involved (69\%). Associated genito-urinary tract anomalies were present in $54 \%$ of cases. Multicystic hypodysplastic, hydronephrotic and segmental macroscopic types of RD were present in 39\%, 23\%, 23\% and $15 \%$ of the cases respectively. The most consistent microscopic feature was primitive ducts surrounded by fibromuscular collarets.

Conclusion: A diagnosis of RD should prompt the search of other associated urinary tract anomalies. Histopathologic examination is required for the definite diagnosis of RD and differentiation from other conditions like polycystic kidney disease, renal hypoplasia, renal atrophy and pure hydronephrosis. This differentiation is important from prognostic and genetic counselling point of view.

Keywords: Multicystic Dysplasia, Renal Dysplasia, Urinary Tract Anomalies.

\section{Introduction}

Renal dysplasia (RD) is a rare congenital disorder culminating from anomalous development of metanephric tissue characterised by disorganised architecture with immature nephrons and undifferentiated stroma. ${ }^{[1,2]}$ It is detected in $2 \%$ of paediatric autopsies with an incidence of one in 3640- 4300 live births..$^{[3,4]}$ It is one of the commonest developmental disorder detected by prenatal ultrasound scan with $77-88 \%$ of the cases detected during third trimester ${ }^{[3,4]} \mathrm{RD}$ is a principal cause of end-stage renal failure in children and its prognosis depends on the quantity of residual functioning renal tissue and presence of other coexisting genitourinary anomalies.$^{[4,5]}$

The aim of the present study was to evaluate the clinicopathologic features of $\mathrm{RD}$ with emphasis on the morphological changes.

\section{Materials and Methods}

This was a single centre retrospective study of cases of $\mathrm{RD}$ diagnosed on nephrectomy specimens over a duration of six years (between August 2011 and August 2017) at M.S Ramaiah Medical College and Hospitals, Bangalore. The clinical details including presentation, age, sex and radiological findings and gross morphological findings were obtained from the patient's case files. The haematoxylin and eosin stained tissue sections were retrieved from the archives and were examined for the various epithelial (primitive glomeruli, primitive ducts and tubular cysts) and mesenchymal (primitive mesenchymal/fibromuscular collarets, metaplastic cartilage, thick walled blood vessels and abundant nerve bundles) microscopic morphological features. On macroscopic morphology RD was classified as: cystic/ multicystic, hypodysplastic, segmental and obstructive. ${ }^{[6,7,8]}$

Statistical Analysis: Data was analysed using Statistical Package for Social Sciences version 20.0, (SPSS, IBM, USA). All the qualitative data was expressed as proportion. The frequency and percentage of each macroscopic type and microscopic features of RD was determined.

\section{Result}

A total of 13 cases of RD were obtained during the six year study period. The age of the cases ranged from 20 weeks of gestation to 54 years, comprising of six adults, six children and one foetus. $8(61 \%)$ of the cases occurred in males and $5(39 \%)$ cases occurred in females with a male: female ratio of 1.6:1.

Clinical Presentation: Five of the cases presented with recurrent urinary tract infection (UTI), four presented as abdominal mass, three presented with renal impairment and hypertension and in one case medical termination of pregnancy (MTP) was done at 20 weeks of gestation 
as the antenatal ultrasound revealed multiple congenital anomalies including left multicystic kidney suggestive of multicystic renal dysplasia.

Ultrasonographic Features: The renal involvement was unilateral in all the cases. The left kidney was involved in $69 \%$ of the cases $(9 / 13)$ and the right kidney in $31 \%(5 / 13)$ of the cases.

The ultrasound findings included cystic renal disease with non-communicating hypoechogenic cysts, hydronephrosis, non-functioning kidney, hydroureter and ureteral duplication, as shown in Table 1. Micturating cystourethrogram (MCU) showed ipsilateral vesico-ureteric reflux (VUR) in two cases.

Associated genito-urinary tract anomalies were present in seven cases (54\%) and included two cases of duplex ureter, two cases of VUR with hydroureter, two cases of ureteropelvic junctional stenosis and one case of undescended testis.

The foetus that underwent MTP, revealed multiple congenital anomalies in other organ systems comprised of cerebral lateral ventriculomegaly with vermian agenesis, cerebellar hypoplasia, tricuspid atresia and single umbilical artery.

Gross Morphologic Features: A varied gross appearance was found. Five cases exhibited multicystic dysplasia (39\%) characterised by small, normal or large sized kidneys with non-reniform nodular external surface and multicystic cut surface (Fig 1). The cysts were non-communicating, randomly oriented and variably sized, filled with clear fluid or hemorrhagic mucoid viscous material. One of the latter cases showed duplex ureter and another case showed hydroureter.

Three cases showed hypoplastic dysplasia (23\%) characterised by small sized kidneys, weighing $<50 \%$ of the normal mean for age. The reniform contour was maintained and the cut surface showed few minute cysts with poor cortico-medullary demarcation. All the cases showed unremarkable pelvis and ureters.

Three cases showed obstructive (hydronephrotic) dysplasia $(23 \%)$ with enlarged kidneys exhibiting single large cystic space surrounded by minimal renal parenchyma. Ureteropelvic junctional stenosis was present in two of the latter cases and in one case hydroureter was present.

Two cases showed segmental dysplasia (15\%) characterised by non-communicating variably sized cysts restricted to the upper pole of kidney. The rest of the renal parenchyma showed reduced renal cortical thickness with preserved cortico-medullary differentiation. Duplex ureter was present in one case.

Microscopic Morphologic Features: The most characteristic epithelial feature that was present in all the cases was primitive ducts lined by undifferentiated to cuboidal epithelium. The most characteristic mesenchymal feature that was present in all the cases was fibromuscular collarets comprised of spindle cells circumferentially arranged around primitive ducts (Fig.2 \& Fig.3). Foci of dystrophic calcification were present in two cases and three cases showed additional findings of chronic pyelonephritis. The other histologic features are summarised in Table 2 (Fig.4 \& Fig.5).

Table 1: Clinical features and ultrasonography findings of renal dysplasia cases.

\begin{tabular}{|c|c|c|l|}
\hline Case No. & Age & Sex & Ultrasound findings \\
\hline 1 & 1year 6 months & Male & Left small kidney with disorganised structure and left undescended testis \\
\hline 2 & 20 weeks gestation & Male & Left kidney with multiple non-communicating hypoechogenic cysts \\
\hline 3 & 32 years & Female & $\begin{array}{l}\text { Ureteral duplication with clustered hypoechogenic cysts in right upper-pole } \\
\text { renal parenchyma }\end{array}$ \\
\hline 4 & 27 years & Male & Right contracted kidney \\
\hline 5 & 7 months & Female & Left hydroureteronephrosis \\
\hline 6 & 2 years & Male & Left multicystic renal dysplasia with ureteral duplication \\
\hline 7 & 20 years & Female & Left contracted kidney \\
\hline 8 & 5 years & Male & Left kidney with multiple non-communicating hypoechogenic cysts \\
\hline 9 & 6 years & Male & Left hydronephrotic kidney \\
\hline 10 & 18 years & Female & Left kidney with multiple non-communicating hypoechogenic cysts \\
\hline 11 & 54 years & Male & Left multicystic kidney with mega ureter \\
\hline 12 & 2 years & Male & Left hydronephrotic kidney \\
\hline 13 & 25 years & Female & Right non-functional kidney \\
\hline
\end{tabular}


Table 2: Microscopic features of renal dysplasia cases.

\begin{tabular}{|l|c|c|}
\hline Histologic features & No. of cases & \% of cases $(\mathbf{n}=\mathbf{1 3})$ \\
\hline Primitive glomeruli & 4 & 30.8 \\
\hline Primitive ducts & 13 & 100 \\
\hline Tubular cysts & 11 & 84.6 \\
\hline $\begin{array}{l}\text { Primitive mesenchymal /fibromuscular } \\
\text { collarets }\end{array}$ & 13 & 100 \\
\hline Cartilage & 5 & 38.5 \\
\hline Thick walled blood vessels & 6 & 46.2 \\
\hline Abundant nerve bundles & 3 & 23.1 \\
\hline
\end{tabular}

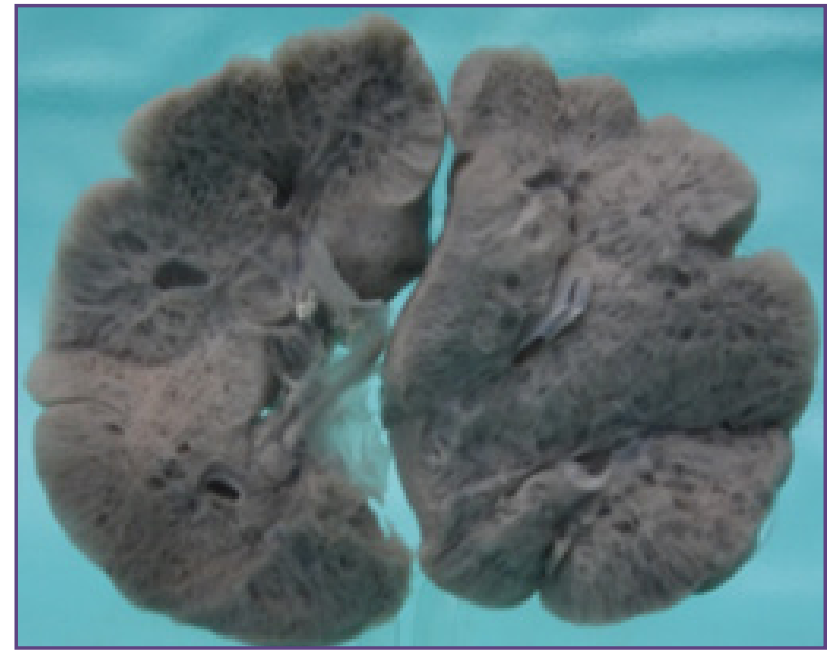

Fig. 1: Multicystic renal dysplasia with kidney exhibiting non-reniform external surface and multicystic cut surface.

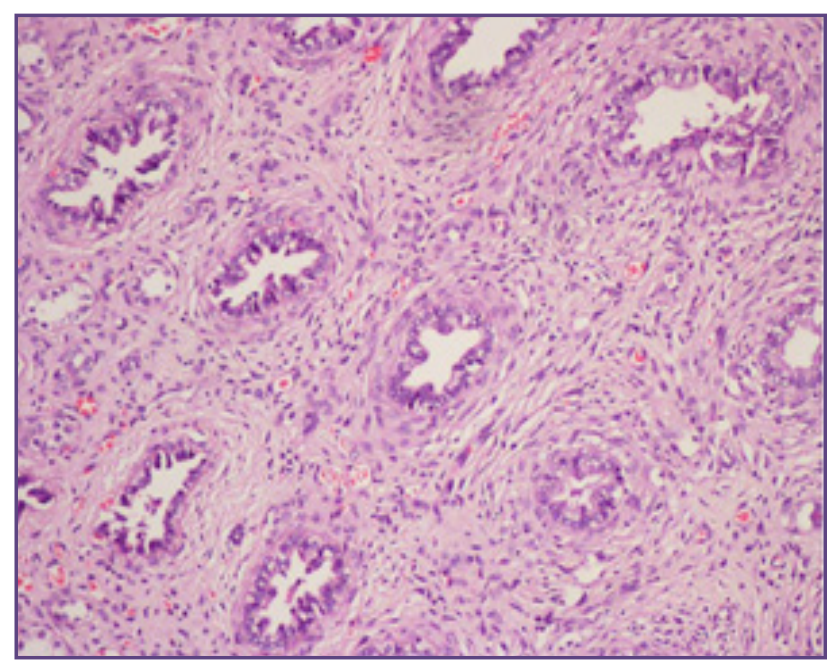

Fig. 3: Renal parenchyma with tubules surrounded by fibromuscular collarets (H\&E, x200).

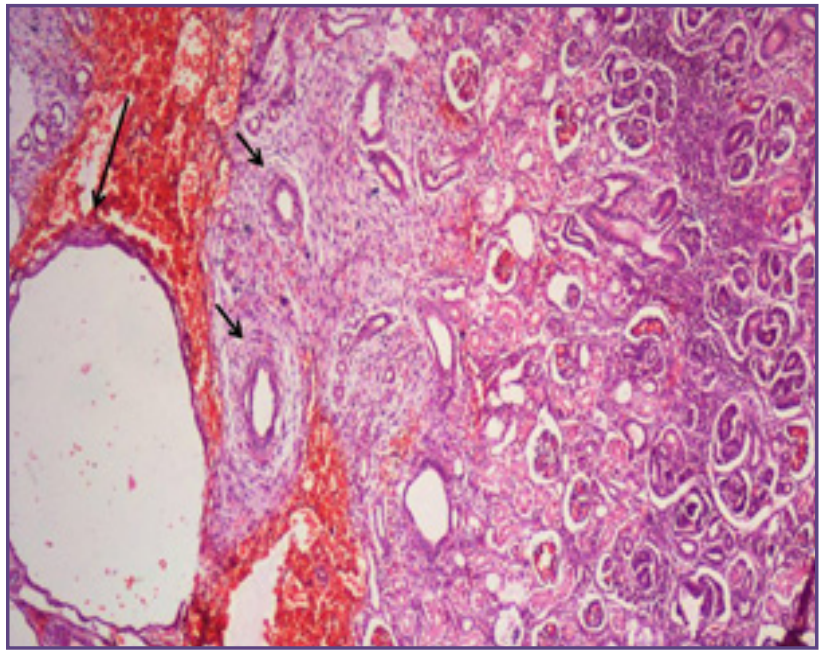

Fig. 2: Renal parenchyma with fibromuscular collarets comprised of spindle cells circumferentially arranged around primitive tubules (small arrows) and tubular cyst lined by flattened epithelium (long arrow) (H\&E, x 100).

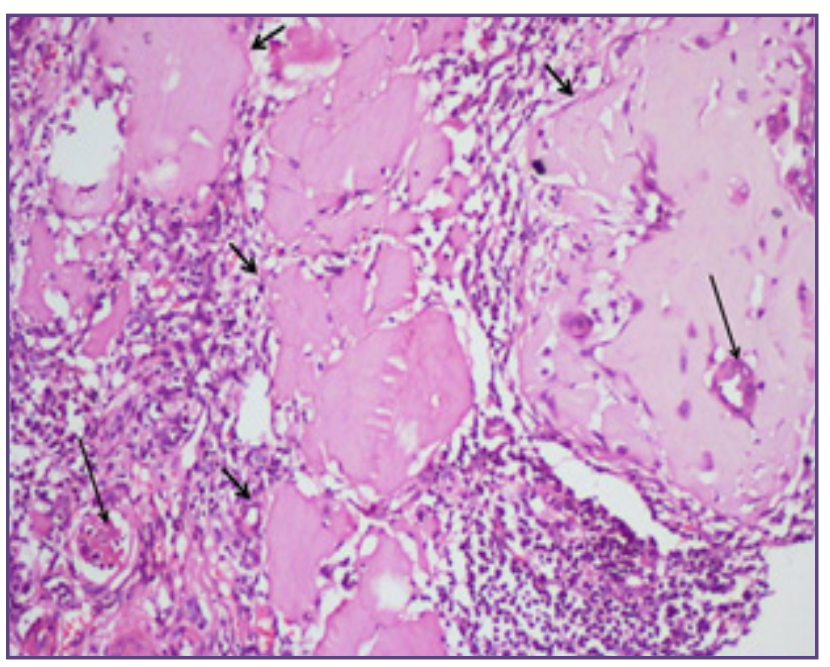

Fig. 4: Dysplastic renal parenchyma with foci of hyaline cartilage (small arrows), primitive tubules ( longer arrows) and chronically inflamed interstitium (H\&E, x200). 


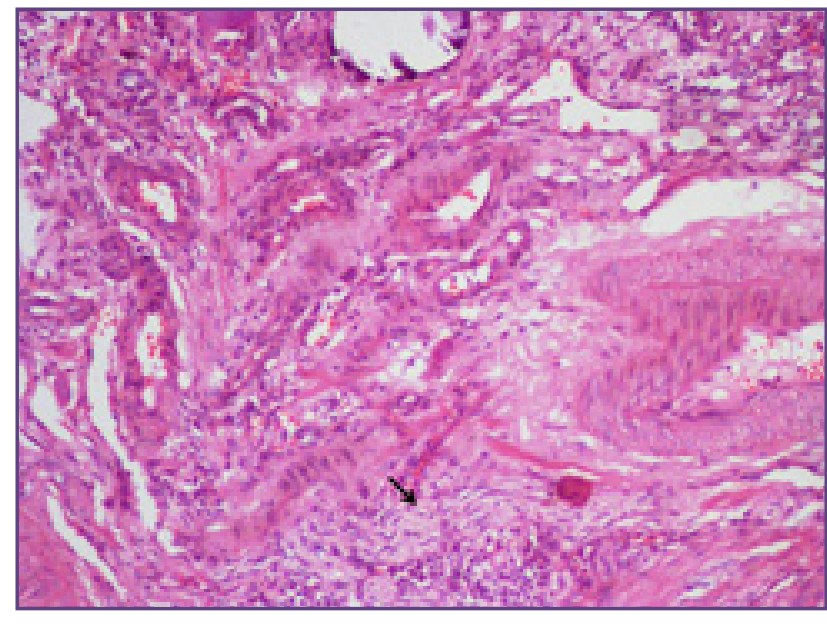

Fig. 5: Dysplastic kidney showing hemangiomatous and thick walled vascular channels and prominent nerve bundles (arrow) (H\&E, x200).

\section{Discussion}

$\mathrm{RD}$ is generally diagnosed in early childhood, however it can be detected by foetal ultrasonography as early as 15 weeks of gestation..$^{[1,9]}$ In a study conducted by Singh et al only one case $(4.5 \%)$ was detected at 21 years and the remaining cases $(95.5 \%)$ were diagnosed from new born period to eight years. ${ }^{[1]}$ In another study all the cases were diagnosed during childhood. ${ }^{[3]}$ However in our study $46 \%$ of the cases were detected in adults, which is a late age for diagnosis. Possibly many of these cases would not have undergone prenatal ultrasound assessment resulting in delayed diagnosis of RD. Further in many of these cases $\mathrm{RD}$ wasn't suspected on imaging and was diagnosed on the nephrectomy specimen which was done for reasons like non-functioning kidney and urinary tract infection. In contrast to western world where more than $80 \%$ of the cases are diagnosed antenatally, large proportion of Indian patients are diagnosed post natally. ${ }^{[10]} \mathrm{A}$ meta-analysis on 67 cohorts with over 3500 patients with unilateral RD revealed male preponderance (59\%) with the left kidney being involved more often than the right kidney. ${ }^{[11]}$ Similar observations were found in our study.

The clinical presentation is wide and depends on the extent and severity of RD and the associated urinary tract anomalies. ${ }^{[1,3,12]}$ In the prenatal age group $\mathrm{RD}$ is always detected on ultrasound imaging as multiple renal cysts or pelvic cysts. ${ }^{[12]}$ In neonates $\mathrm{RD}$ generally presents as palpable flank mass. ${ }^{[12]}$ In children and adults RD presents as UTI, abdominal/flank pain, voiding dysfunction, urinary incontinence, hypertension or impaired renal function. ${ }^{[3,12]}$ In the current study recurrent UTI $(38.5 \%)$ was the commonest presentation followed by abdominal mass
(30.8\%). In one study abdominal mass was the commonest presentation $(66.7 \%)$ and recurrent UTI was present in only $16.6 \%$ of the cases. ${ }^{[3]}$

Renal ultrasonography is the investigation of choice for the initial screening of $\mathrm{RD}$ and also for the evaluation of the contralateral kidney and bladder. ${ }^{[3,12,13]}$ Dimercaptosuccinic acid (DMSA) or technetium Tc 99m mercaptoacetyltriglycine renal scanning may be used to assess the renal function. ${ }^{[1,3]} \mathrm{MCU}$ is used to detect VUR. Imaging modalities like intravenous pyelography, magnetic resonance imaging and magnetic resonance urography are also used. ${ }^{[1,12]}$

The macroscopic appearance of $\mathrm{RD}$ is variable, the malformed kidney may be large and irregular or small and rudimentary or partially or diffusely cystic. ${ }^{[6,12]}$ Different macroscopic types of RD have been described (as mentioned above), based on the extent of dysplasia and cystic components and presence of urine flow obstruction. [6] In our study multicystic dysplasia was the commonest macroscopic type (39\%) followed by hypoplastic dysplasia $(23 \%)$ which is in contrast to studies by Sing et al and Rukmangadha et al where hypoplastc dysplasia was more common followed by multicystic dysplasia. ${ }^{[1,3]}$

A definite diagnosis of renal dysplasia, in the strictest terms, can be made only on histological examination with the most specific features being primitive ducts surrounded by fibromuscular collarets and presence of cartilage. $[1,2,6,12]$. Literature review shows that metaplastic cartilage is present in one third of the cases, similarly in our study cartilage was present in $38.5 \%$ of cases. ${ }^{[6]}$ Cysts were present $84.6 \%$ of our cases which is strikingly similar to the study by Singh et al $(85 \%) .{ }^{[1]}$ These cysts are formed from the primitive ducts and may be large or small, ultimately leading to the heterogenous macroscopic appearance of RD. ${ }^{[12]}$ Primitive glomeruli, thick walled blood vessels and abundant nerve bundles were respectively present in $30.8 \%, 46.2 \%$ and $23.1 \%$ of our cases unlike another study where the later features were present in $100 \%, 100 \%$ and $50 \%$ of the cases. ${ }^{[3]}$

Histopathologic examination is required to differentiate RD from conditions like polycystic kidney disease (PKD), renal hypoplasia and renal atrophy. ${ }^{[12]}$ This distinction is important from the prognostic and genetic counselling point of view. PKD (early onset autosomal dominant PKD and autosomal recessive PKD) may grossly resemble multicystic RD, however cysts are larger and there is no histologic evidence of dysplasia. ${ }^{[2,12]}$ Hypoplastic kidneys exhibit normal reniform shape and are small with reduced 
number of pyramids. Microscopically the nephrons are reduced with normal organisation and there is no evidence of dysplasia. ${ }^{[2,12]}$ Atrophic kidney also lacks dysplasia and exhibits segmental scarring and compensatory hypertrophy of the remaining parenchyma. ${ }^{[12]}$ Hydronephrotic type of $\mathrm{RD}$ has to be differentiated from pure hydronephrosis. In pure hydronephrosis the thinning of the medulla is more extreme than cortex unlike RD where the reverse occurs. ${ }^{[6]}$ Further histological features of dysplasia are not present in pure hydronephrosis.

Associated urinary tract abnormalities (UTA) are said to be present in $\sim 1$ in 3 cases (i.e. $33.3 \%$ ) with unilateral RD, the commonest being VUR. ${ }^{[7]}$ Literature review showed that VUR occurs in $\sim 1$ in 5 cases (i.e. $20 \%$ ) of RD. In the present study VUR was present in $15 \%$ of the RD cases. The other associated UTA include uretero-pelvic junctional stenosis, ureteric duplication, ureterocoele, ureteric ectopia and posterior urethral valves. ${ }^{[1,14]}$ Berna et al retrospectively reviewed 20 cases of unilateral $\mathrm{RD}$ and found concurrent VUR, uretero-pelvic junctional stenosis and duplex system in the contralateral kidney in $15 \%, 15 \%$ and $5 \%$ of the cases respectively. ${ }^{[14]}$ However in our study all the UTA were present in the ipsilateral (i.e. dysplastic) kidney and the above UTA were present in $15 \%, 15 \%$ and $15 \%$ of the cases respectively.

The aetiology of RD is not clear. Potter postulated that $\mathrm{RD}$ is due to a primary defect in ampullary function resulting in diminished branching of ampullary portion of collecting ducts and failure of renal mesenchymal induction.$^{[9]}$ Other theories proposed include foetal urinary obstruction including ureteral atresia, faulty blood supply and nephrogenesis inhibition by unknown substance. ${ }^{[5,15]}$ Knowing that WT1, PAX2/8 and BCL2 genes play an important role in nephrogenesis especially branching morphogenesis and nephron differentiation, it has been proposed that altered expression of these genes results in RD. ${ }^{[12]}$

Prognosis depends on the extent of dysplasia and associated urinary tract anomalies (UTA). RD has been classified as simple and complex by Feldenberg. ${ }^{[1,16]}$ Simple RD is unilateral, without any UTA and has good prognosis with the risk of chronic renal insufficiency or end-stage renal disease at 5 years being nil. ${ }^{[1,10,16]}$ Many of these may completely involute over time. Complex RD is bilateral or is associated with UTA and has a relatively poorer prognosis with high incidence of UTI and renal failure. ${ }^{[1,16]}$ The risk of chronic renal insufficiency and end stage renal disease in complex RD is quoted as $29 \%$ and $21 \%$, respectively. ${ }^{[16]}$ The current trend is conservative management of RD with post-natal ultrasonic surveillance and long term follow up for hypertension, UTI and hyperfiltration injury. ${ }^{[10,12,17]}$ Nephrectomy is done only if the RD is symptomatic. ${ }^{[12]}$

\section{Conclusion}

This study provides information about the clinicopathological features of RD, at a

South Indian tertiary health care centre over a period of 6 years. The clinical presentation, and the frequency and type of associated UTA vary among studies. The commonest presentation in our study was recurrent UTI and coexistent genito-urinary tract anomalies were present in $54 \%$ of cases. Thus, a diagnosis of RD should prompt the search of other associated UTA. The frequent male involvement and left kidney predominance seen in our study corresponds to the pattern described in other studies worldwide. Histopathologic examination is required for the definite diagnosis of RD and differentiation from other congenital/cystic renal conditions. Multicystic RD is the commonest macroscopic type and the most consistent diagnostic microscopic feature is primitive ducts with fibromuscular collarets.

\section{Reference}

1. Sing S, Gupta R, Nigam S, Khurana N, Aggarwal SK, Chaturvedi KU, et al. Clinic-pathological profile of 22 cases of cystic renal dysplasia. Indian J Pathol Microbiol. 2007;50:6-10.

2. Jain S, Liapis H. Dysplasia/Hypoplasia/Agenesis. Chapter 6. In: Colvin RB, editor. Diagnostic Pathology. Kidney diseases. 1st ed. Canada: Amirys Publishing Inc; 2011.p. 6-11.

3. Rukmangadha N, Chowhan AK, Patnayak R, Radhika K, Phaneendra BV, Lakshmi AY, et al. Multicystic dysplastic kidney- a retrospective study with clinicopathological correlation. J Clin Sci Res. 2013;2:88-93.

4. Onal B, Kogan BA. Natural History of Patients With Multicystic Dysplastic Kidney- What Follow up is Needed? The Journal of Urology. 2006;176:1607-11.

5. Woolf AS, Price KL, Scambler PJ, Winyard PJD. Evolving Concepts in Human Renal Dysplasia. JSAN. 2004;15:9981007.

6. Liaps H, Winyard PJD. Cystic Diseases and Developmental Kidney Defects. Chapter 4. In: Jennette JC, Olson JL, Silva FG, D'Agati VD, editors. Hepinstall's Pathology of the Kidney,7th ed. Lippincort Williams \& Wilkins, Philadelphia.2015;1:p.119-171.

7. Bonsib SM. Non-neoplastic diseases of the Kidney. In: Bostwick DG, Cheng L, editors. Urologic Surgical Pathology. 2nd ed. Mosby Elsevier; 2008.p. 11-29.

8. Lin CC, Tsai JD, Sheu JC, Lu HJ, Chang BPH. Segmental multicystic dysplastic kidney in children: clinical presentation, imaging finding, management, and outcome. Journal of Pediatric Surgery.2010;45:1856-62. 
9. Han JH, Lee YS, Kim MJ, Lee MJ, Im YJ, Kim SW, et al. Conservative Management of Segmental Multicystic Dysplastic Kidney in Children. Urology.2015;86:1013-18.

10. Singh JK, Kanojia RP, Narasimhan KL. Multicystic dysplastic kidney in children- a need for conservative and long term approach. Indian J Pediatr. 2009;78:809-12.

11. Schreuder MF, Westland R, van Wilk JA. Unilateral multicystic dysplastic kidney: a meta-analysis of observational studies on the incidence associated urinary tract malformations and the contralateral kidney. Nephrol Dial Transplant. 2009;24:1810-8.

12. Chen RY, Chang H. Renal Dysplasia. Arch Pathol Lab Med. 2015;139:547-51.

13. Lim FF, Tsao TF, Chang HM, Sheu JN. Multicystic Dysplastic Kidney Disease Presenting With a Single Large
Cyst in a Fetus- Anatomical Basis and Radiological Aspects. Pediatrics and Neonatology. 2011;52:227-31.

14. Aytac B, Sehitoglu I, Vuruskan H. Multicystic Dysplastic Kidney: Four-Year Evaluation. Turkish Journal of Pathology. $2011 ; 27: 210-4$.

15. Carmack AJK, Castellan M, Brayfield MP, Gosalbez R. Segmental multicystic dysplasia and ureteropelvic junction obstruction in a nonduplicated kidney. Journal of Pediatric Surgery. 2006;41: E1-E3.

16. Psooy K. Multicystic dysplastic kidney in the neonate: the role of the urologist. Can Urol Assoc J. 2010;4:95-97.

17. Nishio H, Kojima Y, Mizuno K, Kamisawa H, Kohri K, Hayashi Y. Laparoscopic Nephrectomy for Pelvic Multicystic Dysplastic Kidney.

*Corresponding author:

Dr Clement Wilfred D, Associate Professor, Department of Pathology, M.S Ramaiah Medical College and Hospitals, MSRIT Post,

MSR Nagar, Bangalore- 560060 (India)

Phone: +91 9945226314

Email: clement.wilfred@yahoo.com

Financial or other Competing Interests: None. 